\title{
Les enjeux d'une gestion durable de la faune sauvage. La mise en œuvre des ORGFH en France
}

Dealing with sustainable management wildlife. The implementation of the ORGFH in France

\section{Agnès Fortier et Pierre Alphandery}

\section{OpenEdition}

Journals

Édition électronique

URL : http://journals.openedition.org/economierurale/3350

DOI : 10.4000/economierurale.3350

ISSN : 2105-2581

Éditeur

Société Française d'Économie Rurale (SFER)

Édition imprimée

Date de publication : 1 mars 2012

Pagination : 52-64

ISSN : 0013-0559

Référence électronique

Agnès Fortier et Pierre Alphandery, «Les enjeux d'une gestion durable de la faune sauvage. La mise en œuvre des ORGFH en France », Économie rurale [En ligne], 327-328 | janvier-mars 2012, mis en ligne le 01 mars 2014, consulté le 19 avril 2019. URL : http://journals.openedition.org/economierurale/3350 ; DOI : 10.4000/economierurale.3350 


\section{Les enjeux d'une gestion durable de la faune sauvage La mise en œuvre des ORGFH en France}

Agnès FORTIER • INRA, Unité de recherches SADAPT, Systèmes agraires et développement : activités, produits, territoires, Ivry-sur-Seine

fortier@ivry.inra.fr

Pierre ALPHANDERY •INRA, Unité SADAPT, Ivry-sur-Seine

alphande@ivry.inra.fr

$\dot{\mathbf{A}}$ l'heure où la conservation de la biodiversité inspire l'action publique sur la nature $^{1}$, les Orientations régionales de gestion de la faune sauvage et des habitats (ORGFH) viennent témoigner des tentatives de repenser les modalités de gestion de la faune sauvage, en France. La circulaire du ministère de l'Environnement de mai 2002 justifie leur création en ces termes : « $L a$ faune sauvage et ses habitats sont [...] une composante essentielle de notre patrimoine naturel caractérisée par une biodiversité importante. Or la richesse de cette faune sauvage est fortement dépendante des conditions générales de gestion de ses habitats, de la gestion des populations existantes et de la protection des espèces les plus sensibles ».

Gérer durablement la faune sauvage suppose, selon le législateur, de prendre en compte les principales menaces qui pèsent sur celle-ci en lien avec l'évolution des connaissances scientifiques. Les causes majeures de la disparition des espèces ne sont en effet pas tant liées à l'exploitation de certaines d'entre elles (gibier) qu'à la dégradation des milieux dans lesquels elles vivent. L'ambition des ORGFH consiste dès lors à définir les axes d'une politique régionale qui améliore significativement la gestion des habitats, notamment dans les espaces qualifiés d' « ordinaires ». Conçues comme des cadres d'action incitatifs élaborés collec-

1. En particulier, la directive Habitats, promulguée en 1992 par l'Union européenne. Sur ce sujet, voir les travaux du réseau de sociologues Grenat, Pinton et al. (2006). tivement, les ORGFH doivent permettre, à partir d'un état des lieux de la faune sauvage, des habitats et des activités humaines, d'identifier par la concertation les grands enjeux et les propositions de gestion traduites en orientations régionales.

Les ORGFH présentent la singularité d'être issues en 2000 d'une loi portant sur la chasse, ambiguïté institutionnelle qui souligne l'influence mais aussi les controverses attachées en France à cette activité qui joue un rôle politique, économique, écologique et social essentiel dans l'espace rural. Dans un contexte caractérisé par d'importants conflits : contestation virulente de certaines pratiques de chasse, escarmouches récurrentes entre associations écologistes et chasseurs, multiplication des contentieux entre les instances européennes et le gouvernement français, la conservation de la biodiversité constitue l'occasion de redéfinir la légitimité de cette activité pour la rendre socialement acceptable. Le rapport Patriat rédigé préalablement à la loi de 2000 met l'accent sur la nécessaire évolution de la pratique dans le sens d'une responsabilité accrue des chasseurs vis-à-vis du patrimoine commun ${ }^{2}$. "La connaissance scientifique d'aujourd'hui conduit à inventer un nouveau mode de relation des chasseurs à la nature

2. En droit français, la faune sauvage est qualifiée de res nullius (ce qui n'appartient à personne) et donc incompatible avec les objectifs affichés d'une gestion durable. Un projet de loi est en cours qui vise à réformer le statut juridique de la faune sauvage pour le rendre compatible avec le droit européen. 
qui implique, en échange des prélèvements sur la faune, un investissement dans la gestion raisonnée des espèces chassables, dans la régulation des espèces occasionnant des dégâts, dans la protection des habitats. » (Patriat, 2000). En s'attachant à promouvoir le développement durable de la faune sauvage, les pouvoirs publics cherchent en outre à satisfaire aux engagements internationaux de la France impliquée dans plusieurs conventions sur la protection et le maintien de la diversité biologique et, dans le même temps, à légitimer l'activité cynégétique.

La chasse écologique, promue par la loi de 2000, induit des changements significatifs dans les pratiques et la place des institutions cynégétiques, en particulier sur deux points. Elle inscrit la gestion du gibier dans une approche intégrant l'ensemble de la faune sauvage d'une part. Elle requiert de prendre en compte tous les acteurs, gestionnaires et usagers qui, à travers leurs pratiques, ont un impact sur les écosystèmes et les milieux naturels : agriculteurs, sylviculteurs, aménageurs divers, randonneurs, etc., d'autre part. L'analyse de la mise en œuvre des ORGFH constitue dès lors un observatoire privilégié des conceptions et des pratiques des divers groupes sociaux impliqués dans la gestion de la faune et de la manière dont ils s'approprient la référence à la conservation de la biodiversité. Comme on le verra, cette disposition est loin de faire l'unanimité notamment parmi les chasseurs. Nous avons fait l'hypothèse que les difficultés rencontrées par les ORGFH tenaient à ce que l'émergence d'une chasse écologique heurtait les conceptions ancrées depuis plusieurs décennies d'une gestion du gibier organisée autour des seuls chasseurs.

On le voit, l'étude des ORGFH soulève des questions relevant des sociologies rurales, de l'environnement, des sciences et des politiques publiques. Pour mener à bien ces investigations nous avons eu recours à une approche de type qualitative qui combine étroitement l'analyse des textes réglementaires, la réalisation d'entretiens auprès d'acteurs clés du dispositif - à l'échelon national et local - et enfin l'observation participante. La participation aux différentes scènes de débats instituées au niveau national et régional (comité de pilotage, groupe de travail) a constitué une dimension importante de notre analyse. C'est, en effet, au sein de ces espaces de discussion que s'expriment les points de vue ou les controverses sur les modalités de gestion de la faune et de ses habitats. Les résultats présentés ici s'appuient plus particulièrement sur le processus de construction des ORGFH dans trois régions françaises (Haute-Normandie, Franche-Comté et Nord-Pas-de-Calais) et leur mise en perspective avec le travail de synthèse réalisé par l'Office national de la chasse et de la faune sauvage (ONCFS) dans l'ensemble des régions ${ }^{3}$.

Afin de rendre compte du processus d'élaboration des ORGFH et des difficultés auxquelles il s'est trouvé confronté, on s'attachera dans une première partie à mettre en perspective le modèle de gestion écologique de la chasse avec celui de la gestion rationnelle du gibier à l'œuvre depuis cinquante ans. La seconde partie sera consacrée à la mise en œuvre des ORGFH. Nous montrerons qu'en dépit de l'hostilité affichée envers cette disposition par la nouvelle majorité politique arrivée au pouvoir en 2002, le travail d'élaboration des ORGFH verra le jour tout en donnant lieu à de nombreuses controverses. Enfin, l'examen de différentes études de cas dans la dernière partie sera l'occasion de souligner que le processus d'élaboration des ORGFH dépend aussi des particularités régionales.

3. Le ministère de l'Écologie et du Développement durable (MEDD) a délégué à l'ONCFS le soin de réaliser à intervalles réguliers une synthèse de l'état d'avancement des travaux dans les différentes régions. Les rencontres organisées à l'échelon national et les documents établis par l'ONCFS nous ont ainsi permis de confronter nos données et d'enrichir notre analyse. 


\section{De la gestion rationnelle du gibier à la gestion écologique de la faune sauvage}

Les années 1970 marquent un tournant dans l'histoire de la chasse en France. La conception de l'activité cynégétique fondée jusqu'alors sur une logique de prélèvement est supplantée peu à peu par un modèle gestionnaire axé sur la préservation du « capital » gibier (Colin, 1985). Cette volonté de moderniser la chasse dans le sens d'une gestion rationnelle intervient dans un contexte de profondes transformations (Chamboredon, 1982 ; Dalla Bernardina, 1983 ; Fabiani, 1988 ; Darbon, 1997 ; Traïni, 2003).

- Transformations induites par la modernisation de l'agriculture, tout d'abord. Celleci s'accompagne d'un cortège de mesures (remembrement, développement de la monoculture, utilisation croissante de machines, d'engrais, de pesticides) qui conduit à un appauvrissement des habitats et donc à une réduction des populations du gibier de plaine ;

- par la diversification des modes de recrutement (les chasseurs issus de la paysannerie sont de moins en moins nombreux) conjuguée à une diversification des usages de l'espace rural induisent des modifications dans les représentations associées à la pratique cynégétique ;

- à travers l'émergence d'une sensibilité écologiste centrée sur l'état de la ressource, la stigmatisation du chasseur « viandart » et une évolution des rapports à l'animal.

La nécessité de promouvoir une nouvelle éthique de la chasse fondée sur le respect de l'équilibre entre les activités et le caractère sportif et désintéressé de la pratique (Fabiani, 1982) s'impose alors aux responsables cynégétiques, d'autant plus que la pression de la chasse est importante. L'effectif des chasseurs ne cesse, en effet, de croître entre 1960 et 1975 et aggrave le phénomène de raréfaction du gibier. Les actions engagées se situent donc à un double niveau : favoriser la reproduction de la faune chassable d'une part et « réguler » la population de chasseurs d'autre part.

L'un des principaux outils de gestion alors utilisé pour limiter les prélèvements de gibier est le plan de chasse. Institué en 1966 pour certaines espèces de grands gibiers et rendu obligatoire en 1979, ce dispositif réglementaire a pour objectif de parvenir à un équilibre agro-sylvo-cynégétique, autrement dit de rendre compatible la présence de certains gibiers avec les activités agricoles et forestières. À ce titre, il prévoit un système de quota visant à limiter le prélèvement des animaux sur un territoire donné, en fonction de sa capacité d'accueil. Ce dispositif, fondé sur le comptage et le tir sélectif, s'accompagne d'un ensemble de mesures destinées également à favoriser les conditions de reproduction de la faune chassable : agrainage, cultures à gibier, entretien du milieu, protection contre les prédateurs, efforts de repeuplement (lâchers), etc. La loi de 1968 sur l'indemnisation des dégâts causés par le sanglier et le grand gibier aux récoltes ${ }^{4}$, constitue une étape supplémentaire dans la responsabilité accordée aux chasseurs dans la gestion de la faune sauvage. Ces dispositions marquent l'avènement d'une période où la chasse apparaît de plus en plus comme un loisir « moderne » qui tend à se dissocier de la paysannerie, elle-même en pleine transformation. Le chasseur s'autonomise à travers un ensemble de pratiques qui lui confère le statut de « producteur de gibier» (Traïni, op. cit.).

Cette influence prépondérante du monde cynégétique trouve aussi sa traduction dans le mode particulier d'organisation de la chasse en France. La gestion de l'activité, d'abord dévolue au ministère de l'Agricul-

4. Celle-ci stipule que les agriculteurs renoncent au droit de défendre leur culture contre la faune (droit d'affût) en échange d'une indemnité versée par les instances cynégétiques en cas de dégâts de gibier. 
ture, puis transférée en 1971 au ministère de l'Environnement, lors de sa création, a été en réalité confiée par l'administration à des organismes intermédiaires. L'État a en effet délégué une grande partie de ses prérogatives à des structures associatives, organisées selon un modèle fédéral et réputées très influentes en raison de leurs capacités financière, organisationnelle et politique. Dans cette configuration, les Fédérations départementales de chasse (FDC) ont acquis un véritable monopole de la gestion de l'activité (Darbon, op. cit., Traïni, op. cit.). À titre d'exemple, ce sont elles qui, sous le contrôle du préfet, lequel se montre le plus souvent bienveillant, élaborent les plans de chasse avec le concours de l'administration décentralisée du ministère de l'Agriculture. Ce système, malgré les critiques venues notamment des rangs des mouvements écologistes (associatifs et politiques), s'est perpétué jusqu'à la fin des années 1990 , entérinant tacitement une répartition des responsabilités. La gestion de la nature « ordinaire » a été confiée aux chasseurs, aux agriculteurs et aux forestiers tandis que les espaces et les espèces qualifiés de remarquables relevaient de l'intervention des naturalistes.

La loi chasse de 2000 qui crée les ORGFH remet en cause ce partage en se fondant sur une approche qui intègre la pluralité des gestionnaires et des usagers concernés et ce, sur l'ensemble du territoire. La gestion durable de la faune sauvage requiert en effet de ne pas se cantonner à la gestion des prélèvements mais implique de garantir les conditions de «production » de la ressource, en associant l'ensemble des gestionnaires du milieu : agriculteurs, sylviculteurs, randonneurs, aménageurs et autres usagers de l'espace. L'un des principaux enjeux de cette réforme consiste, en effet, à intégrer les objectifs de maintien, voire de restauration de la diversité biologique dans les processus de production agricole et sylvicole et dans les projets d'aménagement, autrement dit de promouvoir des pratiques et des modes de gestion compatibles avec les exigences de la faune sauvage. Par ailleurs, la distinction opérée entre espèces chassables et espèces remarquables est elle aussi remise en question au profit d'une approche qui prend en compte l'ensemble de la faune sauvage et inclut désormais la faune «banale » et ses habitats dits « ordinaires » qui couvrent la grande majorité du territoire. Cette nouvelle exigence contribue à brouiller les catégories en présence et oblige les chasseurs et les environnementalistes, dont les rapports sont le plus souvent conflictuels, à trouver des compromis. Le rapport Patriat insiste sur la nécessité d'un tel rapprochement. «Il n'y aura pas de chasse d'avenir sans ouverture d'un dialogue entre les parties concernées. Les quelques responsables qui refusent tout dialogue prennent le risque de conduire à l'extinction de la chasse. » (Patriat, op. cit.)

En définitive, la chasse écologique promue par la loi de 2000 modifie les pratiques et la place des institutions cynégétiques dans le sens d'une réduction de l'autonomie des chasseurs. Cette évolution va de pair avec une réorganisation institutionnelle voulue par le législateur en vue d'une clarification des compétences respectives des fédérations et de l'administration. L'Office national de la chasse (ONC), établissement public à caractère administratif, rebaptisé Office national de la chasse et de la faune sauvage (ONCFS), n'est plus « sous contrôle » des fédérations de chasse, mais placé sous la double tutelle du ministère de l'Agriculture et de l'Environnement. Outre la redéfinition de ses missions axées désormais sur « la mise en valeur et la surveillance de la faune sauvage » et le « respect de la réglementation en matière de police de la chasse », son conseil d'administration comprend en plus des représentants de l'État et des chasseurs, des experts de la faune sauvage et de la protection de la nature. Les fédérations départementales de chasse conservent toutefois une mission de service public. Elles participent « à la mise 
en œuvre du patrimoine cynégétique départemental et à la protection de la faune sauvage et de ses habitats ». Dans ce cadre, l'État leur délègue la mise en place des Schémas départementaux de gestion cynégétique (SDGC) (largement inspirés des plans de chasse) qui, selon le texte de loi, devront être établis en conformité avec les ORGFH. L'élaboration de ce nouveau cadre de décision à l'échelle des régions, conçu selon une dynamique de type partenariale, confirme que la gestion durable de la chasse et de la faune sauvage ne peut plus être l'apanage des seuls chasseurs, mais repose sur l'ensemble des gestionnaires et des usagers de l'espace, y compris les environnementalistes. L'intégration du droit de chasse dans le droit de l'environnement ${ }^{5}$, qui constitue également un des objectifs de cette loi, témoigne là encore de la volonté de faire évoluer la chasse dans le sens d'une meilleure prise en compte de la biodiversité et du développement durable, sans pour autant faire l'unanimité parmi les chasseurs.

\section{ORGFH \\ La place de la chasse en question}

Promulguée sous un gouvernement de gauche par D. Voynet, alors ministre de l'Environnement, jugée hostile au monde de la chasse, la loi de 2000 est remise en cause à l'occasion du changement politique qui intervient deux ans plus tard. Conformément à ses promesses électorales, la nouvelle majorité désormais ancrée à droite revient sur les dispositions majeures de la loi et introduit une série de réformes, en 2003, à l'occasion de la nouvelle loi chasse, puis en 2005, dans le cadre de la loi sur le développement des territoires ruraux (DTR). Dans un discours prononcé à l'Assemblée nationale en février 2003, la ministre de l'Environnement légitime l'autonomie restituée aux chasseurs dans la gestion de leur

5. Par l'inclusion de ses dispositions dans le code de l'environnement et non plus dans le code rural. activité par l'importance de leur place au sein du monde rural. "Les chasseurs se sont préoccupés les premiers de la disparition des zones humides, de la préservation des haies et des aménagements pour la faune. Je n'ose pas imaginer ce que seraient les équilibres naturels sans eux [...]. Qui prendrait en charge le coût de la protection, celui de la réparation des dégâts ? » Et de rappeler en conclusion de son discours : "Les chasseurs assurent le maintien de la biodiversité et de l'équilibre du monde rural. Dans un monde qui se déshumanise, ils sont les gardiens de l'identité de territoires menacés $»^{6}$. Par cette déclaration, la ministre tente de fonder la légitimité de la chasse sur des considérations sociales et identitaires sans abandonner la référence à la conservation de la biodiversité devenue incontournable. Officiellement, les ORGFH ne sont pas supprimées, mais leur portée est remise en cause et leur avenir demeurera flou jusqu'en 2005.

C'est dans ce contexte d'incertitude suscité par les débats parlementaires sur la loi DTR que le ministère de l'Environnement décide de lancer, en 2003, la procédure d'élaboration des ORGFH. Ce dernier souhaite agir rapidement étant donné les risques de remise en cause de cette mesure par la nouvelle majorité, dans le cadre de la préparation de la loi DTR. Dans la circulaire du 3 mai 2002, le ministère de l'Environnement précise que le déroulement des ORGFH devra comporter trois grandes étapes.

- Tout d'abord, la réalisation d'un état des lieux régional de la faune sauvage et de ses habitats qui précisera l'impact des activités humaines sur les espèces et les milieux. - Ensuite, la définition des grands enjeux régionaux et les objectifs associés.

- Enfin, leurs déclinaisons sous forme de mesures.

6. Déclaration de Madame Bachelot, le 26 mars 2003. 
Placée sous la responsabilité des Directions régionales de l'environnement (Diren) et réalisée conjointement avec le soutien technique des délégations régionales de l'ONCFS, la procédure d'élaboration des ORGFH est conçue comme un projet territorial mené en concertation avec l'ensemble des acteurs concernés. Ce sont ces derniers qui doivent définir collectivement les enjeux et les mesures adaptées à la situation régionale. Selon un responsable de l'ONCFS, le choix de cette procédure centrée sur la définition et la résolution de problèmes locaux ainsi que l'implication d'un large panel d'acteurs devrait permettre de sortir de l'opposition chasseurs/protecteurs de la nature qui prévaut à l'échelle nationale. Plus fondamentalement, les conditions de mise en œuvre de cette action publique sont ici essentielles. Il s'agit moins de parvenir à des résultats concrets que de favoriser l'émergence d'un climat de confiance, le rapprochement des acteurs en présence, conditions indispensables pour permettre l'appropriation des objectifs contenus dans les ORGFH. C'est dans cette optique qu'il faut interpréter le caractère peu prescriptif de ces orientations qui ont avant tout une visée prospective. La circulaire du 3 mai 2002 rappelle en effet que «si les orientations régionales n'ont pas vocation à constituer des programmes opérationnels, elles doivent cependant inciter les différents partenaires concernés à engager des programmes d'action qui s'avéreront utiles pour la faune sauvage et ses habitats ».

L'élaboration des ORGFH s'est opérée dans le cadre de ce que l'on peut qualifier de « dispositif », notion que nous définissons à la suite de Marc Mormont comme un ensemble composé «d'arrangements institutionnels mettant en relation des représentations, des normes, des pratiques, des espaces et des acteurs » (Mormont 1996). L'observation de ce dispositif et l'analyse des débats auxquels il a donné lieu dans trois régions, conjugué à un état des lieux du déroulement des ORGFH dans l'ensemble des régions de France (document de travail réalisé par l'ONCFS, 22 mars 2004), ont révélé la faible implication d'un certain nombre d'acteurs dont la profession agricole, les élus locaux ainsi qu'une partie des chasseurs et des naturalistes. Ils ont aussi montré la difficulté à dépasser les clivages entre chasseurs et protecteurs de la nature. La question de la place de la chasse s'est en effet avérée centrale et a cristallisé de nombreux débats. Les critiques les plus virulentes sont venues des rangs naturalistes. France Nature Environnement (FNE) a appelé à un boycott des ORGFH pour marquer son mécontentement face à un ensemble de décisions qui, selon elle, favorisaient outrageusement les chasseurs. «Les ORGFH, c'est une opération politique $d u$ monde de la chasse pour essayer de déstabiliser le monde naturaliste », précisait l'un des responsables du dossier à FNE. " Il s'agit de placer les chasseurs sur l'ensemble du territoire pour s'occuper de l'ensemble des espèces. » Cette crainte de voir les chasseurs utiliser la légitimation écologique de la loi de 2000 pour déborder leur domaine habituel s'est aussi fondée sur le virage environnemental opéré par une partie des fédérations de chasse. Celles-ci ont en effet acheté et géré des espaces naturels, effectuant ainsi des opérations de conservation de milieux fragiles en partenariat avec des collectivités locales ou organisant la collecte de données sur des espèces qui ne les intéressaient pas jusqu'alors. Pour autant, tous les naturalistes n'ont pas adopté une posture de boycott. Certains dirigeants, comme ceux de Haute-Normandie Nature Environnement (HNNE), ont choisi de se servir des ORGFH comme d'une tribune pour dénoncer la place laissée par l'État aux chasseurs. «Les ORGFH ne sont qu'un habillage alors que seul le dossier chasse importe. Ce qui se cache est la volonté du monde de la chasse de devenir gestionnaire de l'espace. On va leur donner un rôle d'interlocuteur de l'État pour l'espace; les citoyens sont écartés et ne sont qu'une 
caution dans cette affaire. " D'autres, au contraire, comme la Ligue pour la protection des oiseaux (LPO) de Franche-Comté ou le Groupe ornithologique et naturaliste du Nord (GON) qui revendiquaient leurs compétences scientifiques, ont estimé qu'il fallait saisir l'opportunité des ORGFH pour travailler avec de nouveaux partenaires. Ainsi, les naturalistes - on utilise également en France le terme équivoque d'écologiste (Alphandéry et al., 1991) - ont, suivant les cas, participé ou pas à l'élaboration des ORGFH. La diversité de leurs positions a reflété d'ailleurs largement l'hétérogénéité d'un milieu associatif souvent fragmenté en petites structures.

Du côté des chasseurs, l'évolution vers une gestion écologique de la faune n'a pas fait l'unanimité. Là encore, on a pu observer des positions contrastées selon les fédérations de chasse qu'on peut analyser dans le prolongement des réactions suscitées par la directive Oiseaux de 1979, aboutissant à la création du mouvement Chasse pêche nature et tradition (CPNT) (Darbon, op. cit.). Beaucoup de chasseurs se disaient hostiles aux ORGFH qui faisaient entrer la chasse dans le champ des questions environnementales et remettaient en cause leur maîtrise de l'organisation de la pratique cynégétique. D'où la réticence d'une partie d'entre eux à s'impliquer dans l'élaboration des ORGFH. Selon un responsable de FRC, « le monde de la chasse est d'abord fédéral. Ce qui signifie qu'on va se faire imposer comment gérer nos espèces et nos habitats par une démarche régionale, autrement dit une certaine vision de l'environnement $»$.

Les relations entre naturalistes et chasseurs se sont souvent avérées délicates car les ORGFH venaient signifier combien leurs domaines d'activité étaient susceptibles de se rencontrer, en dépit de références éthiques (Larrère, 2004) et de conceptions de la gestion de la nature très différentes. Les chasseurs ont privilégié une démarche par les milieux ou les habitats alors que les naturalistes mettaient plus volontiers en avant une entrée par les espèces. On pourrait considérer qu'il s'agit d'approches complémentaires, si une longue histoire conflictuelle ne venait envenimer les relations. De ce fait, les naturalistes ont fréquemment dénoncé la tendance des chasseurs à investir des domaines nouveaux alors qu'ils n'en avaient pas la compétence, voire la légitimité (ils étaient soupçonnés de ne s'intéresser qu'au gibier).

À l'inverse, certains chasseurs, à l'image de ce responsable implanté en FrancheComté, ont mis aussi en doute la compétence des naturalistes, leur reprochant une vision statique en termes de protection des espèces et de sanctuarisation des espaces au détriment d'une vision pragmatique, dynamique et pluraliste. Ils ont assuré que leur présence sur le terrain et leurs moyens financiers leur permettaient de s'appuyer sur des réseaux plus efficients que ceux des naturalistes. Mais les querelles entre chasseurs et naturalistes se sont aussi focalisées sur la production de connaissances qui constitue un enjeu majeur depuis que les « défenseurs des oiseaux » ont obtenu gains de cause auprès de la cour de justice européenne en mobilisant des arguments scientifiques pour réduire la période de chasse des migrateurs (Alphandéry, Fortier, 2007). À l'occasion d'une table ronde réunissant le monde de la chasse, les scientifiques et les principaux représentants de l'espace rural $^{7}$, les responsables institutionnels cynégétiques des régions Nord-Pas-de-Calais et Picardie, ont tour à tour dénoncé la suprématie des savoirs scientifiques comparé à d'autres types de connaissances plus à même de rendre compte de la complexité et de la variété des contextes locaux. "Nul n'a le monopole de la science » faisait remarquer l'un d'eux, «il

7. Organisée en janvier 2003, lors de la consultation nationale sur la Charte de l'environnement. Cette dernière, préparée par le ministère de l'Environnement, visait à inscrire dans la Constitution un certain nombre de principes destinés à favoriser la prise en compte de l'environnement et du développement durable. 
n'y a pas une vérité scientifique » constatait un second et les autres de surenchérir : «On s'appuie sur des rapports d'experts non objectifs »; "C'est un problème d'honnêteté intellectuelle. Les scientifiques sont souvent des anti-chasse ». Ces désaccords illustrent plus fondamentalement l'affrontement entre deux modèles d'expertises. Le premier, fondé sur une approche positive de la science, est défendu par de nombreuses associations de protection de la nature qui considèrent que seules les connaissances validées par les scientifiques sont légitimes pour assurer le bien-fondé de la décision. Ce modèle est directement à mettre en relation avec le processus de «scientifisation » de la politique décrit par Habermas dans les années 1960 (Habermas, 1978). Le second est qualifié par Hermitte et Roqueplo (1997) de modèle procédural. Il est basé sur trois grands principes : le contradictoire (qui permet l'expression des différentes thèses en présence), la transparence, l'indépendance, et présente la particularité de combiner des connaissances scientifiques et des savoirs d'action. Au-delà des divergences observées entre chasseurs et naturalistes à propos de l'élaboration des ORGFH, se pose bien évidemment la question de la place respective des uns et des autres dans la gestion de la faune sauvage.

Les clivages entre chasseurs et naturalistes sont apparus plus prégnants du fait de l'absentéisme d'un certain nombre d'acteurs. Nos enquêtes, combinées aux travaux de synthèse effectués par l'ONCFS dans les différentes régions, ont en effet fait ressortir que les conditions de la confrontation d'une pluralité de points de vue sur la faune n'ont pas été réunies dans tous les dispositifs régionaux. La difficulté à impliquer un certain nombre de partenaires dont la profession agricole, les élus locaux a tenu d'abord au caractère incertain, conflictuel et peu prescriptif des ORGFH. Mais elle a résulté également des ressentiments accumulés dans les campagnes françaises par ceux qui critiquaient les politiques de la nature (Alphandéry, 2001). Ainsi, certains agriculteurs se sont déclarés échaudés par l'absence de moyens financiers, consacrés aux mesures agri-environnementales ou au réseau Natura 2000, et se sont montrés se sont montrés hostiles au modèle de gestion écologique de la faune sauvage qui stigmatisait les effets ravageurs pour les habitats de plusieurs décennies d'une intensification de la production non maîtrisée. Dans ces conditions, le processus de concertation a souvent relevé davantage d'une recherche de consensus que d'un véritable débat approfondi entre l'ensemble des partenaires représentés. D'autant que les Diren ont accueilli ce dossier avec une certaine réserve.

«La plupart [...] ne s'étaient pas encore sorties de Natura 2000 quand elles ont vu arriver les ORGFH. N'étant guère présentes et légitimes sur le plan de la faune sauvage, elles se sont méfiées de cette "patate chaude" rendue explosive par les problèmes de la chasse. »

(Naturaliste responsable d'un bureau d'études.)

Des espaces de discussion plus ou moins formels (comité de pilotage, groupes de travail techniques ou thématiques) ont été mis en place, mais avec le souci d'éviter les sujets par trop polémiques sur lesquels des désaccords profonds existaient, en particulier entre chasseurs et naturalistes. Néanmoins, on a pu constater, dans un certain nombre de cas, qu'il était possible de définir un cadre commun permettant l'expression et la confrontation d'acteurs dans des configurations très diverses, comme l'ont montré les observations réalisées dans trois régions françaises.

\section{Diversités régionales}

- L'élaboration des ORGFH de HauteNormandie a mis en présence la principale association naturaliste de la région, hostile à la chasse, et des institutions cynégétiques très impliquées dans le processus (à l'exception des chasseurs de gibier d'eau) et soucieuses de tenir compte des ORGFH dans la rédaction des SDGC. 
Cependant, comme nous l'avons vu plus haut, le dirigeant de cette association ne croit pas en la possibilité d'un débat public et constructif : «La Diren négocie en coulisses avec les chasseurs. Ceux-ci ne sont pas des gestionnaires de la nature : seul le gibier les intéresse. C'est l'État qui doit être garant de l'aménagement de l'espace. Mais les naturalistes n'ont pas le pouvoir de lobbying obtenu par les chasseurs grâce au vote ; il n'existe pas de vote ciblé sur la chasse ». Ce refus du principe du partenariat avec des institutions cynégétiques s'est situé délibérément sur le plan politique plus que sur le terrain de la gestion de la nature. Du côté des autres acteurs impliqués, les élus locaux de même que la profession agricole ont peu participé alors que les forestiers ont été davantage présents, mais sans illusion sur la capacité de la procédure à briser le monopole des chasseurs. En revanche, les pratiques agricoles très intensives ont été pour la première fois évoquées (et donc validées) dans le document cadre des ORGFH comme une cause majeure de destruction des habitats. Ce qui a donné lieu à une série de préconisations (changements d'itinéraires techniques, maintien, voire recréation «d'éléments fixes $^{8}$ dans le paysage ») sans caractère obligatoire, mais qui tendent à montrer que la gestion de la nature centrée sur les espèces patrimoniales cède peu à peu du terrain au profit d'une approche relevant de l'écologie fonctionnelle.

- En Franche-Comté, la plupart des acteurs ont affirmé être en accord avec l'approche développée par les ORGFH. Selon un responsable de la LPO régionale, «les ORGFH ont eu le mérite de changer les choses dans les têtes. Elles ont aussi permis d'affiner une méthodologie et donné une vision à moyen terme à beaucoup d'acteurs, notamment aux collectivités locales.

8. Ceux-ci désignent les arbres isolés, haies, bosquets, ripisylves, mares.
Je suis content que notre association n'ait pas suivi l'appel au boycott de FNE». Cependant, l'échec de la constitution d'un réseau régional de production de données sur la faune vertébrée a aussi montré à quel point les rapprochements entre naturalistes et chasseurs étaient difficiles. $\mathrm{La}$ LPO, liée à l'Université, a joué la carte de la compétence scientifique et s'est dite favorable à «la professionnalisation des activités d'expertise » pour valoriser des savoir-faire que les chasseurs n'avaient pas. Dans le même temps, les naturalistes redoutaient la multiplication des initiatives prises par les chasseurs en matière de gestion des milieux, tendance symbolisée par le nom donné au siège de la fédération des chasseurs du Jura, la «Maison de la nature et de la faune sauvage ». Les chasseurs s'estimaient, en effet, légitimes à intervenir comme experts sur l'ensemble de la faune sauvage. Selon le président de la fédération départementale des chasseurs du Jura, « il ne doit pas y avoir de chasse gardée en matière de faune sauvage », tout en se montrant critique vis-à-vis des approches naturalistes menées en termes d'espèces prioritaires : «Les catégories adoptées pour caractériser les espaces ou les espèces sont des normes qui figent les situations ». L'attitude de la Diren a consisté à renvoyer les protagonistes dos-à-dos. «Les chasseurs ont beaucoup de moyens et sont incontournables [précise le responsable en charge du dossier]. Soit les naturalistes s'associent aux chasseurs, soit ils disparaîtront ; à eux de se défendre. L'un de leurs atouts est de produire des publications. »En définitive, le cas de la Franche-Comté illustre le fait que les approches différentes des naturalistes et des chasseurs, lorsqu'elles se déploient sur des espaces communs, les transforment en concurrents plutôt qu'en partenaires. 
- Dans la région Nord-Pas-de-Calais, les naturalistes et les chasseurs, malgré des rapports tendus, ont décidé de collaborer dans le cadre d'un Comité technique dont le rôle a consisté à proposer des méthodes de travail à un Comité de pilotage à la composition élargie. Comme dans plusieurs autres régions françaises, la fédération régionale des chasseurs a été chargée par la Diren de réaliser un état des lieux par grands types d'habitats tandis que les membres du GON étaient désignés comme responsables du recensement des espèces non chassables. Les agriculteurs représentants la FNSEA sont venus jouer les trouble-fête dans ce dispositif, refusant de valider la procédure choisie pour élaborer les ORGFH, de crainte qu'elle ne devienne contraignante pour leurs pratiques culturales, à l'image du règlement d'éco-conditionnalité qui accompagne depuis peu la politique agricole commune de l'UE. Le Comité technique a donc revu la procédure, en y associant étroitement les agriculteurs et les forestiers. Les ORGFH ont été élaborées à l'issue d'âpres négociations. Le consensus qu'elles ont révélé se fondait sur l'implication des différents acteurs concernés et sur un partage des rôles clairement défini entre chasseurs et naturalistes : les chasseurs s'occupent de tout ce qui a trait au gibier et les naturalistes du reste de la faune sauvage. Les instances cynégétiques ont par ailleurs fait valoir leurs compétences et les ressources dont elles disposaient en matière de données sur la faune sauvage. Lors du Comité de pilotage de restitution des ORGFH qui réunissait l'ensemble des protagonistes, le président de la fédération des chasseurs du Nord a exprimé son intention de créer un Observatoire régional de la faune chassable susceptible d'associer les forestiers. Ce réseau serait ainsi établi en parallèle avec celui des naturalistes. La situation en région Nord-Pas-
de-Calais se démarque ainsi des cas étudiés précédemment en ce sens que la plupart des acteurs ont souhaité être impliqués dans la démarche, sans pour autant s'inscrire dans un seul et unique réseau.

Ces situations diverses expliquent la difficulté de tirer un bilan global du processus d'élaboration des ORGFH. En dépit des limites qu'elle a comportées, l'adoption d'une démarche de type concertée a permis à un certain nombre d'acteurs et d'institutions qui cherchaient à se positionner sur les nouveaux enjeux de conservation de la biodiversité de se rencontrer, de confronter leurs savoirs et leurs connaissances sur les espèces. Elle a en outre favorisé la construction de cadres de référence autour de la gestion de la faune sauvage, mettant en relation espaces, espèces et pratiques humaines, opérant des classifications et construisant des catégories. Selon un bilan dressé par l'ONCFS, les pistes d'action ont concerné en priorité la préservation des habitats (47\%), la gestion et la conservation des espèces $(33 \%)$, enfin, l'amélioration et la diffusion des connaissances (15\%) (Wencel, 2006). Reste à savoir ce qu'il adviendra de ces propositions. Dans quelle mesure les ORGFH seront-elles davantage qu'un catalogue d'intentions et déboucheront-elles sur la réalisation d'actions concrètes ? La traduction des ORGFH en actions autour des thèmes majeurs que constitue par exemple « la restauration de la capacité d'accueil des habitats » ou « la fragmentation des milieux » suppose que se construisent des liens de coopération et d'apprentissage mutuel entre les acteurs concernés. Les conflits que nous avons évoqués rendent souvent difficile ce «passage à l'action » qui a comme préalable l'existence de visées communes et la reconnaissance de la légitimité des pratiques et des savoir-faire des autres. 


\section{Conclusion}

Le suivi de l'élaboration concertée des ORGFH révèle avant tout la difficulté à mobiliser et à faire dialoguer des acteurs et des institutions essentiels dans la gestion de l'espace et de la faune comme les représentants de la profession agricole, les élus, certains chasseurs et naturalistes. Ces difficultés illustrent combien la gestion durable de la faune sauvage est porteuse d'enjeux importants aux yeux des protagonistes. Pour les agriculteurs, il s'agit d'une mesure supplémentaire destinée à modifier leurs pratiques dans le sens d'une prise en compte des habitats. Pour les naturalistes, cette disposition apparaît souvent comme une opportunité offerte au milieu cynégétique d'étendre ses prérogatives à l'ensemble du territoire et de la faune sauvage. Côté chasseurs enfin, l'intégration de la chasse dans les questions environnementales a pour conséquence de marquer davantage leur interdépendance avec les autres acteurs et usagers de l'espace et donc de remettre en cause leur autonomie dans la gestion de la pratique cynégétique. De plus, force est d'admettre que les positions sont loin d'être homogènes au sein de ces groupes, notamment parmi les chasseurs et les naturalistes. Le dispositif de concertation adopté dans le cadre des ORGFH nous a en effet permis d'identifier une gamme de rapports différenciés entre chasseurs et naturalistes d'une région à l'autre, allant du refus de collaborer pour des raisons d'ordre éthique (posant la question de la légitimité de la chasse) à la nécessité de dialoguer et d'envisager diverses formes de partenariat. Cependant, le fait que le modèle de gestion écologique ne soit pas parvenu à s'imposer au sein du monde de la chasse n'implique pas pour autant que les institutions qui le régissent se désintéressent de la préservation de la biodiversité. On peut, en effet, constater qu'en maints endroits, les chasseurs se sont approprié une partie des conceptions portées par la gestion écologique à travers la multiplication des partenariats et des initiatives en faveur de la conservation des habitats. Cette dynamique correspond à une volonté des dirigeants de redonner une légitimité à la pratique cynégétique et de rompre avec la logique de «forteresse assiégée ».

Si les résultats observés ne sont pas spectaculaires et si les réalisations collectives procèdent par petites touches, la gestion écologique de la chasse s'impose peu à peu comme un cadre d'action tout en suscitant des tensions et des contradictions. La succession des réformes engagées à la suite de la loi chasse de 2000, en 2003 puis en 2005 et qui a conduit à limiter la portée des ORGFH, a confirmé le poids politique des chasseurs dans la gestion de la faune sauvage en France. Ces lois successives ont aussi été l'occasion de remodeler l'image de la chasse en affichant sur des modes divers son rôle essentiel sur le plan social et économique en milieu rural. Ces conceptions de la pratique cynégétique renvoient à des univers distincts comme le montre le contenu hétéroclite de la loi DTR : celle des campagnes françaises du productivisme agricole triomphant où la gestion de la faune appartenait de plein droit à la chasse et celle plus récente inspirée par le référentiel de la conservation de la biodiversité. Cette double filiation ne doit pas faire oublier que la chasse ne saurait être réductible à la seule gestion écologique mais est aussi l'expression de divers modes d'appartenances sociales et territoriales en particulier dans les sociétés de chasse dites «populaires » (Baticle, 2007 ; Raison du Cleuziou, 2008).

De Natura 2000 aux ORGFH en passant par la Stratégie nationale pour la biodiversité, la succession des actions publiques affine par agrégations successives les connaissances et les outils consacrés à la conservation de la biodiversité. L'élaboration de ces orientations a notamment permis de mettre en évidence le caractère lacunaire et difficilement mobilisable des 
connaissances relatives à la faune sauvage. Cela a amené les responsables des ORGFH de plusieurs régions à envisager la création d'Observatoires régionaux de la faune sauvage. Initiatives qui recoupent les ambitions affichées par la Stratégie nationale de la biodiversité de développer des systèmes d'information régionaux du patrimoine naturel, qualifiés aujourd'hui d'Observatoires de la biodiversité. De plus, la nécessité de penser étroitement la faune sauvage en rapport avec les habitats a conduit à mobiliser d'autres types de savoirs relevant de l'écologie fonctionnelle, comme la dynamique des populations ou l'écologie du paysage. Le recours aux notions de " réseaux », de corridors écologiques censés favoriser la circulation ou le déplacement des espèces en est une illustration. À travers l'objectif affiché de certaines mesures de restaurer des connexions endommagées par la pression anthropique (développement des transports, intensification agricole), les ORGFH préfigurent le projet de Trame verte et bleue nationale institué à l'occasion du Grenelle de l'environnement. Au-delà des enjeux scientifiques, la mise en place de ces réseaux écologiques offre l'opportunité à des acteurs qui s'ignorent où se côtoient sans véritablement se connaître, de dialoguer pour repenser ensemble leur territoire. Nul doute que cela ne se fera pas sans difficultés, tant sont diverses les représentations et les usages de la nature et de l'espace portés par les acteurs qui cohabitent dans les communes rurales. À cet égard, l'exemple des ORGFH vient rappeler que l'écologisation des pratiques est loin d'être un processus linéaire. 


\section{RÉFÉRENCES BIBLIOGRAPHIQUES}

Alphandéry P., Bitoun P., Dupont Y. (1991). L'équivoque écologique. La Découverte. Alphandéry P. (2001). Les campagnes françaises de l'agriculture à l'environnement (1945-2000) : politiques publiques, dynamiques sociales et enjeux territoriaux. Thèse de doctorat, Sociologie-Fondation Nationale des Sciences Politiques (FNSP), Institut d'études politiques de Paris, $465 \mathrm{p}$.

Alphandery P., Fortier A. (2007). A new approach to wildlife management in France. The formulation of the ORGFSH as tools for the conservation of biodiversity. Sociologia Ruralis, vol. 47, $\mathrm{n}^{\circ} 1$, p. 42-62.

Baticle C. (2007). Les pratiques de chasse comme affirmations politiques du principe d'autochtonie : dimensions territoriales des luttes cynégétiques. Thèse de doctorat de sociologie et d'anthropologie, Université de Picardie-Jules Verne, Amiens, 3 vol., 910 p.

Chamboredon J.-C. (1982). La diffusion de la chasse et la transformation des usages sociaux de l'espace rural. Études Rurales, n ${ }^{\circ} 87-88$, p. 233-260.

Colin G. (1985). Rapport sur la modernisation du droit de la chasse et de la faune sauvage. Ministère de l'Environnement.

Dalla Bernardina S. (1983). La nature sauvage et ses consommateurs. Des stéréotypes du récit de chasse aux lieux communs de la prose écologiste. Thèse de doctorat d'Ethnologie, Université de Provence, Aix-Marseille 1.

Darbon D. (1997). La crise de la chasse en France. L'Harmattan.

Dodier N. (2001). Les leçons politiques de l'épidémie de Sida. Éditions de l'EHESS.

Fabiani J.-L. (1988). Les prédateurs éclairés : remarques sur la gestion rationnelle de la chasse en France. In « L'imaginaire de la chasse », Atelier CRC, France, p. 111-124.
Fabiani J.-L. (1982). Quand la chasse populaire devient un sport. La redéfinition sociale d'un loisir traditionnel. Études Rurales, ${ }^{\circ} 87-88$, p. 309-323.

Habermas J. (1978). La technique et la science comme idéologie. Gallimard.

Hermitte M.-A. (1997). L'expertise scientifique à finalité politique. Réflexions sur l'organisation et la responsabilité des experts. Justices, $\mathrm{n}^{\circ}$ 8, p. 79-103.

Larrère R. (2004). Les conflits entre les chasseurs et les protecteurs de la nature. La Ricerca folklorica, p. 111-117.

Mormont M. (1996). Agriculture et environnement : pour une sociologie des dispositifs. Économie rurale, nov-déc.

Patriat F. (2000). Propositions pour une chasse responsable et apaisée. Rapport au Premier ministre, La Documentation Française.

Pinton F. (coord.), Alphandéry P., Billaud J.-P., Deverre C., Fortier A., Gesniaux G., Lefebvre C. (coll.) (2006). La construction du réseau Natura 2000 en France. Paris, La Documentation française, $254 \mathrm{p}$.

Roqueplo P. (1997). Entre savoir et décision, l'expertise scientifique. Paris, INRA.

Raison Du Cleuziou Y. (2008). De la résistance à la subversion. Les chasseurs de la baie de Somme et le développement durable. Études Rurales, vol. 1, n ${ }^{\circ} 181$, p. 133-148.

Traïni C. (2003). Les braconniers de la République. Puf.

Trivière F.-X. (2002). La chasse dans l'estuaire de la Loire. Mission du patrimoine ethnologique, ministère de la Culture, doc. Ronéo. 61 p.

Wencel M.-C. (2006). Les ORGFH : deux années de concertation en faveur de la nature ordinaire. Faune sauvage, $\mathrm{n}^{\circ} 270$, janvier. 